\title{
TITLE:
}

\section{Above-threshold ionization by chirped laser pulses}

$\operatorname{AUTHOR}(S)$ :

Nakajima, T

\section{CITATION:}

Nakajima, T. Above-threshold ionization by chirped laser pulses.

PHYSICAL REVIEW A 2007, 75(5): 053409.

ISSUE DATE:

2007-05

URL:

http://hdl.handle.net/2433/50423

RIGHT:

Copyright 2007 American Physical Society 
PHYSICAL REVIEW A 75, 053409 (2007)

\title{
Above-threshold ionization by chirped laser pulses
}

\author{
Takashi Nakajima* \\ Institute of Advanced Energy, Kyoto University, Gokasho, Uji, Kyoto 611-0011, Japan
}

(Received 3 April 2007; published 16 May 2007)

\begin{abstract}
We theoretically investigate above-threshold ionization by chirped laser pulses. By comparing the photoelectron energy spectra and the photoelectron angular distributions of $\mathrm{Na}$ for the laser pulses with different chirp rates but with the identical spectral profile, we find that the ionization processes have a clear dependence on the chirp rate. Further calculations without excited bound states during the time propagation of the wave function reveal practically no chirp dependence, which is clear evidence that the origin of the chirp dependence in above-threshold ionization is the excited bound states.
\end{abstract}

DOI: 10.1103/PhysRevA.75.053409

PACS number(s): $32.80 . \mathrm{Rm}, 42.50 . \mathrm{Hz}$

\section{INTRODUCTION}

When the laser intensity is sufficiently high, atoms can absorb more than the minimum number of photons required to ionize. This is the so-called above-threshold ionization (ATI) and, after the first experimental observation [1], there are many experimental as well as theoretical works reported in the literature [2]. In most of the studies, however, transform-limited pulses are employed, and there is no detailed study for the chirped pulses. If the intensity is so high that tunneling ionization or barrier suppression ionization takes place effects of the chirp may not be very important, since it is the field that induces ionization from the ground state. Actually this is the reason why a simple tunneling model turned out to be quite successful to explain the main features, at least qualitatively, of the dynamics occurring in the tunneling ionization regime. On the other hand, if the intensity is lower, it would be multiphoton ionization that mainly contributes to the ionization process where, in principle, the chirp may play a more important role, since ionization takes place through near-resonant intermediate states. Indeed, in a recent paper [3], we have theoretically clarified that the origin of the subpeaks in the ATI spectra of Mg is the far off-resonantly excited bound state(s) by the spectral wing of the transform-limited pulse. However, study on the effects of chirped laser pulses remains intact in our previous study.

Chirped laser pulses can be an interesting tool to probe and control the ultrafast dynamics at any laser intensities. Actually there are a variety of studies reported in the literature under different intensity regions. For the low intensity region, there are some reports in which few-level atoms have been employed to study and control the dynamics with chirped laser pulses $[4,5]$. For the moderate intensity region a semi-nonperturbative analysis has been carried out [6] for the $\mathrm{Cs}$ atom with a few bound states and the continuum. For the higher intensity region, there are several reports for molecules where understanding the photoionization process and the bondlength dynamics [7-9] as well as dissociative ionization $[10,11]$ are the main interest. The behavior of atoms exposed to chirped laser pulses in the moderate to high intensity regions still remains an open question.

In this paper we theoretically investigate the effects of the chirp of ultrafast laser pulses on the ATI processes of an

*Email address: t-nakajima@iae.kyoto-u.ac.jp atomic system. The time-dependent Schrödinger equation (TDSE) is our workhorse and $\mathrm{Na}$ is chosen as a target atom. In order to make a meaningful comparison, we have chosen the laser pulses to have the identical spectral profile but with different chirp rates. As we will show later on in this paper, we find a clear chirp dependence for the photoelectron angular distribution (PAD) and the photoelectron energy spectra (PES): The PES exhibit some structure for the chirped pulses compared with the transform-limited pulses. Consistently the PADs are different for the different chirp rates. Moreover, even if the magnitude of the chirp rate is chosen to be the same, the results turn out to be different for the different directions (positive or negative) of the chirp. In contrast, results of the TDSE calculations without excited bound states show practically no dependence on the chirp for both PES and PADs. This is clear evidence that the chirp dependence comes from the excited bound states. Physically this means that, even if the spectral profile of the laser pulses is identical, the excitation and accordingly ionization dynamics critically depend on which bound states are excited by how much during the chirped pulse.

\section{TIME-DEPENDENT SCHRÖDINGER EQUATION}

In order to study the interaction of $\mathrm{Na}$ with chirped fs laser pulses, we employ the TDSE on the discretized atomic basis states constructed in a spherical box. As we have already shown in our recent paper [3], time propagation of the total wave function on the atomic basis states $[12,13]$ enables us to pinpoint the physical origin of the dynamics of interest $[3,14,15]$ which is actually the main advantage of this method compared with other methods which propagate the numerical wave function itself [17]. The level structure of $\mathrm{Na}$ is shown in Fig. 1. The $\mathrm{Na}$ atom has only one valence electron outside the closed shell and the ionization potential is rather low, i.e., $5.14 \mathrm{eV}$ from the ground state. In contrast, the ionization potential of $\mathrm{Na}^{+}$lies $47.3 \mathrm{eV}$ above that of $\mathrm{Na}$. Therefore, we may safely neglect the core excitation at the intensities of $\sim 10^{12} \mathrm{~W} / \mathrm{cm}^{2}$, which means that only the valence electron will play an essential role during the interaction with the laser pulse. All the atomic basis functions we need, $\phi_{n l m}(\boldsymbol{r})$ where $n, l$, and $m$ are the principal, orbital, and magnetic quantum numbers, respectively, are constructed using the Hartree-Fock potential with a correction term to effectively take into account core polarization. Briefly, by ex- 


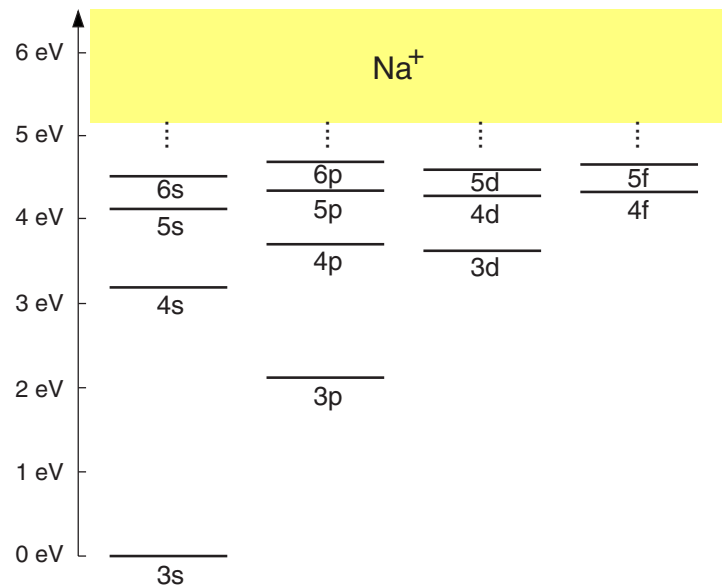

FIG. 1. (Color online) Relevant energy levels of Na.

panding the atomic basis states with a set of $B$-spline functions, the time-independent Schrödinger equation can be reduced to the eigenvalue problem [16], from which we obtain the eigenenergies and eigenfunctions.

Now we expand the total wave function, $\Psi(t)$, on the basis functions [12], $\phi_{n l m}(\boldsymbol{r})$, with time-dependent coefficients, $b_{n l m}(t)$, i.e.,

$$
\Psi(\boldsymbol{r}, t)=\sum_{n, l, m} b_{n l m}(t) \phi_{n l m}(\boldsymbol{r}),
$$

which satisfies the TDSE,

$$
i \frac{\partial \Psi(\boldsymbol{r}, t)}{\partial t}=\left[H_{0}+V(t)\right] \Psi(\boldsymbol{r}, t),
$$

where $H_{0}$ is the field-free atomic Hamiltonian for the $\mathrm{Na}$ atom and $V(t)$ the time-dependent interaction between the atom and the laser pulse, which is, using the dipole approximation in the velocity gauge, defined by $V(t)=-\mathbf{A}(t) \cdot \mathbf{p}$ with $\mathbf{A}(t)$ being the vector potential of the laser field and $\mathbf{p}$ the momentum of the valence electron. By assuming that the field is linearly polarized along the $z$ direction and has a Gaussian temporal envelope, the vector potential of the linearly chirped pulse can be defined in the form of

$$
\boldsymbol{A}(t)=\hat{z} A_{0} \exp \left[-i(\omega t+\phi)-4 \ln 2\left(\frac{t}{\tau_{\mathrm{TL}}}\right)^{2}\left(\frac{1}{1-i \xi}\right)\right],
$$

where $A_{0}$ is the peak value of the vector potential, $\phi$ the carrier-envelope phase, $\tau_{\mathrm{TL}}$ the pulse duration [full width at half maximum (FWHM)] for the transform-limited pulse, and $\omega$ the central photon energy at $t=0 . \xi$ stands for the dimensionless linear chirp rate and we can easily show that the pulse duration (FWHM) of the chirped pulse, $\tau_{\mathrm{FWHM}}$, is connected to the pulse duration of the transform-limited pulse, $\tau_{\mathrm{TL}}$, by $\tau_{\mathrm{FWHM}}=\tau_{\mathrm{TL}} \sqrt{1+\xi^{2}}$. The positive (negative) values of $\xi$ means that the instantaneous laser frequency increases (decreases) with time. Obviously $\xi= \pm \sqrt{3}$ and $\pm \sqrt{15}$ correspond to the linearly chirped pulses with $\tau_{\mathrm{FWHM}}=2 \tau_{\mathrm{TL}}$ and $4 \tau_{\mathrm{TL}}$, respectively. Note that the use of the above definition for the vector potential guarantees that the spectral profile of the chirped pulse with any $\xi$ is always identical to that of the transform-limited pulse with the duration of $\tau_{\mathrm{TL}}$.

By substituting Eq. (1) into Eq. (2) it is clear that Eq. (2) can be recast into a set of first order ordinary differential equations for the time-dependent coefficients, $b_{n l m}(t)$, with the initial condition that $\Psi(\mathbf{r}, t=-\infty)=\phi_{3 s 0}(\mathbf{r})$. After solving the TDSE, we can compute the PES and the PADs from the relations of

$$
\frac{d P}{d \epsilon}=\sum_{n(\epsilon>0) l m}\left|b_{n l m}(t)\right|^{2}
$$

and

$$
\left.S(\theta, \epsilon) \equiv \frac{d P}{d \theta}\right|_{\epsilon}=\left|\sum_{l m}(-1)^{l} e^{i \delta_{l}(\epsilon)} \sqrt{2 l+1} P_{l}(\cos \theta) b_{n l m}(t)\right|^{2},
$$

where $P$ and $\epsilon$ represent the photoelectron yield and its energy, respectively, $\delta_{l}(\epsilon)$ is a sum of the phase shift due to the Coulomb and non-Coulomb potentials, and $P_{l}$ are the Legendre polynomials. Since we have constructed a set of atomic basis states for the bound as well as continuum states, it is straightforward to extract the phase shift of the continuum wave function at a given energy. Note that the timedependent coefficients, $b_{n l m}(t)$, become constant after the pulse since we employ the interaction picture to solve Eq. (2).

\section{NUMERICAL RESULTS AND DISCUSSION}

For the numerical calculations we have chosen the photon energy and the peak intensity to be $1.55 \mathrm{eV}$ at $t=0$ corresponding to the Ti:sapphire lasers and $I=10^{12} \mathrm{~W} / \mathrm{cm}^{2}$, respectively, with and without the chirp, unless otherwise noted. The corresponding Keldysh parameter, $\gamma$, is 6.6 for $\mathrm{Na}$ under these laser parameters. The carrier-envelope phase, $\phi$ in Eq. (3), has been set to 0 for all numerical calculations since the pulse duration we assume in this paper is more than a few cycles and accordingly there are no notable phasedependent effects. As we can see in Fig. $1,4 s, 6 p$, and $5 f$ states, etc., would play important roles since they are close to resonance at this photon energy. The pulse duration of the transform-limited pulse, $\tau_{\mathrm{TL}}$, is set to be 13.3 fs, i.e., 5 optical cycles. The chirped pulses we have employed have the duration of $\tau_{\mathrm{FWHM}}=2 \tau_{\mathrm{TL}}$ (for $\xi= \pm \sqrt{3}$ ) and $4 \tau_{\mathrm{TL}}$ (for $\xi$ $= \pm \sqrt{15}$ ). Note that the chirped pulses have exactly the same spectral profile with that of the transform-limited pulse. The atomic basis states we need to solve the TDSE are constructed in a rather large box size so that the reflection of photoelectrons with high kinetic energies affects neither PES nor PADs. After several computer runs, we have found that the box radius of 2400 (atomic units) with the number of $1602 B$-splines of order 9 and the number of orbital angular momenta from $L=0$ up to $L=8$ gives a reasonable convergence for the most demanding case in this paper, i.e., chirped pulse with $\tau_{\mathrm{FWHM}}=4 \tau_{\mathrm{TL}}$.

Figure 2 shows the PES for the transform-limited pulses with different durations, $\tau_{\mathrm{TL}}=13.3 \mathrm{fs}$ ( 5 cycles), $26.6 \mathrm{fs}$ (10 


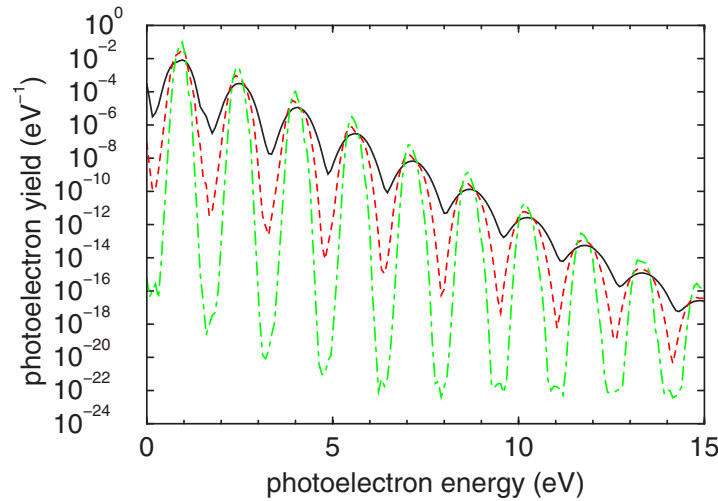

FIG. 2. (Color online) Photoelectron energy spectra by the transform-limited pulses at the photon energy of $1.55 \mathrm{eV}$ with the durations of $\tau_{\mathrm{TL}}=13.3 \mathrm{fs}$ (solid), $26.6 \mathrm{fs}$ (dashed), and $53.2 \mathrm{fs}$ (dot-dashed).

cycles), and 53.2 fs ( 20 cycles). Naturally the widths of the ATI peaks for the shorter pulse duration are broader, reflecting the broader spectral bandwidth of the laser pulses. The height of each ATI peak is higher for the longer pulse simply because we have assumed that all pulses have the same peak intensity.

In Fig. 3 we show the PES for the chirped pulses. Clearly the chirp rate, $\xi$, strongly affects the PES, which we attribute to the different contribution of intermediate bound states for the different chirp rates. Namely, depending on the value of $\xi$, some bound states close to the multiphoton resonance are in real excitation, and as a result strongly affected by the time-dependent laser frequency. Recall that the real excitation of the states requires a finite time while the virtual excitation can be instantaneous. As a result, excitation dynamics of the intermediate bound states can be very different, depending on how the instantaneous frequency component of the chirped pulse changes in time. This also explains why the PES is different for the different signs of $\xi$ with the same $|\xi|$. Corresponding to the different orders of the ATI peaks labeled as $S=2,4,6$, and 8 in Fig. 3, the PADs are shown in Figs. 4(a)-4(d). Note that, for all chirped pulses, the photo-

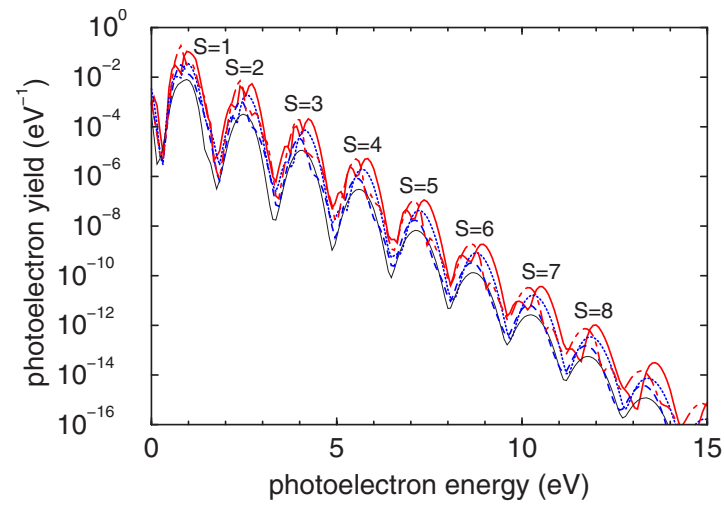

FIG. 3. (Color online) Photoelectron energy spectra by the chirped pulses with the chirp rates of $\xi=-\sqrt{15}$ (solid), $\xi=-\sqrt{3}$ (dotted), $\xi=0$ (thin solid), $\xi=\sqrt{3}$ (dashed), and $\xi=\sqrt{15}$ (dot-dashed). The photon energy is $1.55 \mathrm{eV}$ and $\tau_{\mathrm{TL}}=13.3 \mathrm{fs}$ for the transform-limited pulse.

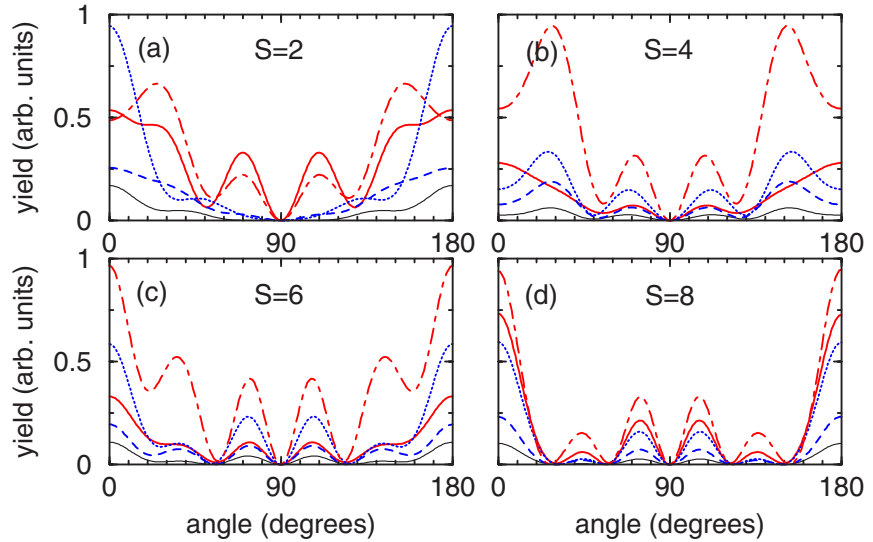

FIG. 4. (Color online) Photoelectron angular distributions at the different photoelectron energies by the chirped pulses with the chirp rates of $\xi=-\sqrt{15}$ (solid), $\xi=-\sqrt{3}$ (dotted), $\xi=0$ (thin solid), $\xi=\sqrt{3}$ (dashed), and $\xi=\sqrt{15}$ (dot-dashed). The orders of the ATI peaks are (a) $S=2$, (b) $S=4$, (c) $S=6$, and (d) $S=8$, as labeled in Fig. 3. The photon energy is $1.55 \mathrm{eV}$ and $\tau_{\mathrm{TL}}=13.3 \mathrm{fs}$ for the transform-limited pulse.

electron energies to be employed for the PADs have been chosen at the positions of the peaks for the transform-limited pulse in Fig. 3, since the PES by the chirped pulses have some structures. Clearly the PADs exhibit an even clearer chirp dependence than the PES.

In order to verify our interpretation that the chirp dependence essentially comes from the intermediate bound states, we have performed additional computer runs under a fictitious condition; we have solved the TDSE after the removal of the excited bound states from the atomic basis set. Note that this is one of the main advantages of expanding the total wave function in terms of the atomic basis states. Figures 5 and 6 are the results for the PES and the PADs, respectively. Clearly the PES does not show the chirp dependence. The only difference we see in Fig. 5 is that the heights of the ATI peaks are different for the different chirp rates. This, however, is simply due to the fact that we have employed the same peak intensity for the all chirped pulses. Therefore, after the appropriate normalization, the difference of the PES in Fig. 5 practically disappears. Consistently, the PADs in

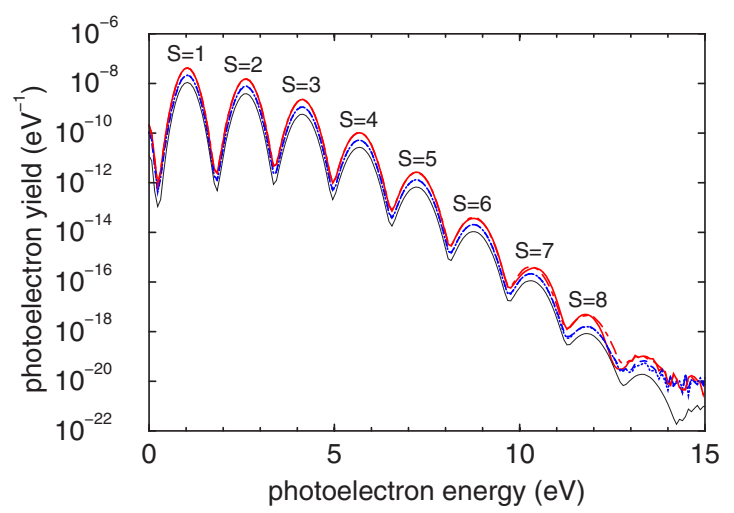

FIG. 5. (Color online) Same as Fig. 3 but the time propagation has been performed without the excited bound states. After the appropriate normalization all the PES become practically identical. 


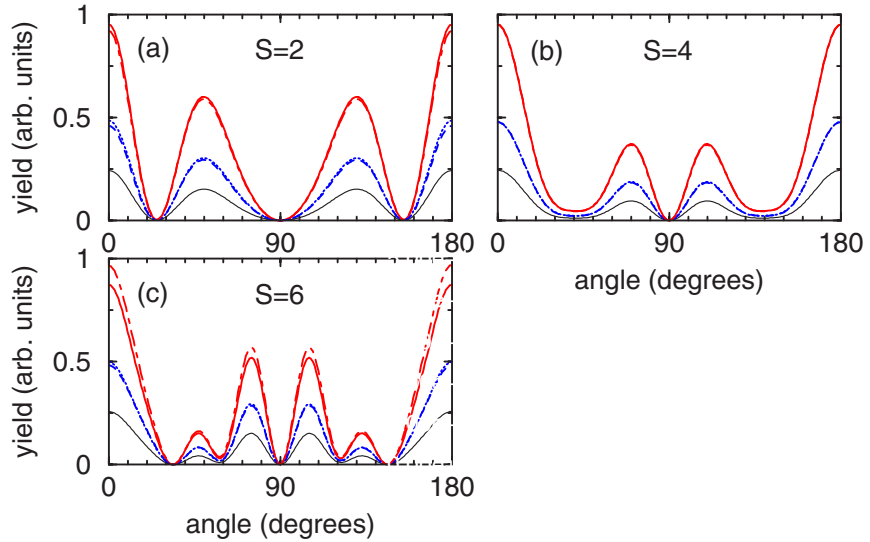

FIG. 6. (Color online) Same as Fig. 4 but the time propagation has been performed without the excited bound states. After the appropriate normalization all the PADs in each panel become practically identical.

Figs. 6(a)-6(c) hardly exhibit the chirp dependence. Again, after the appropriate normalization, the PADs become almost identical for the different chirp rates.

As a further confirmation, we have calculated the PES and PADs for the photon energy of $6.2 \mathrm{eV}$ at $t=0$ so that the single-photon absorption already goes beyond the ionization threshold. This is a realistic way of avoiding the influence of the intermediate bound states. The results are shown in Figs. 7 and 8 for the PES and PADs, respectively. The dip located at $1.2 \mathrm{eV}$ of Fig. 7 is a Cooper minimum [18]. It is again clear that the chirp dependence is seen in neither PES nor PADs, which is consistent with our interpretation about the role of the intermediate bound states for the chirped laser pulses.

Before closing this section, we would like to note that the above interpretation would be valid in the multiphoton ionization regime, since it is known that the intermediate bound states play important roles in this intensity range. A natural question would be how it would be if the intensity is higher and tunneling ionization is the dominant ionization process. We naively expect that the chirp plays a lesser role in the

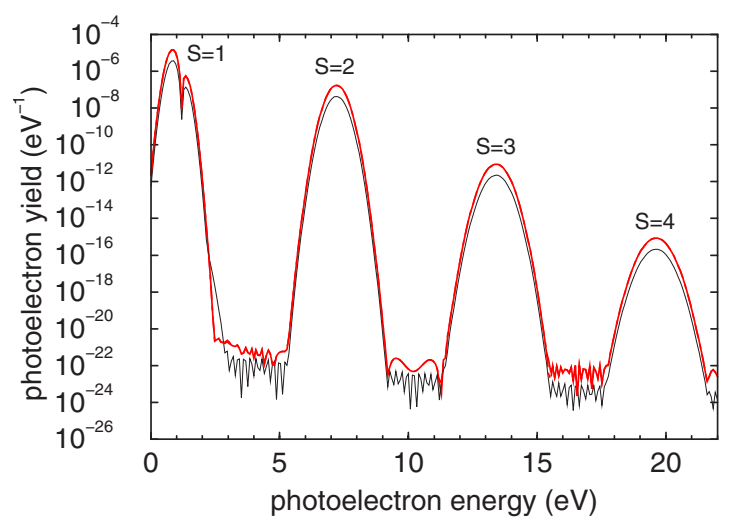

FIG. 7. (Color online) Photoelectron energy spectra by the chirped pulses with the chirp rates of $\xi=-\sqrt{15}$ (solid), $\xi=0$ (thin solid), and $\xi=\sqrt{15}$ (dot-dashed). The photon energy is $6.2 \mathrm{eV}$ and $\tau_{\mathrm{TL}}=6.65 \mathrm{fs}$ for the transform-limited pulse. After the appropriate normalization all the PES become practically identical.
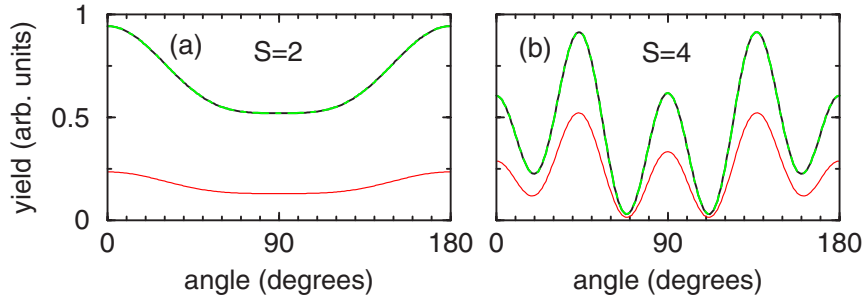

FIG. 8. (Color online) Photoelectron angular distributions at the different photoelectron energies by the chirped pulses with the chirp rates of $\xi=-\sqrt{15}$ (solid), $\xi=0$ (thin solid), and $\xi=\sqrt{15}$ (dot-dashed). The orders of the ATI peaks are (a) $S=2$ and (b) $S=4$ as labeled in Fig. 7. The photon energy is $6.2 \mathrm{eV}$ and $\tau_{\mathrm{TL}}=6.65 \mathrm{fs}$ for the transform-limited pulse. After the appropriate normalization all the PADs in each panel become practically identical.

tunneling ionization regime than that in the multiphoton ionization regime because excited bound states would play a lesser role. However, further study would be necessary to confirm our argument. Indeed a lot of things, which are not taken into account in the above simplified argument, can happen, and the numerical calculation becomes much more demanding at the higher intensity range. The answer may not be so simple.

\section{CONCLUSIONS}

In conclusion we have theoretically studied abovethreshold ionization of $\mathrm{Na}$ by chirped laser pulses. We have found that both photoelectron energy spectra and photoelectron angular distributions are strongly dependent on the chirp rate even if the spectral profile of the laser pulses are chosen to be the same. In contrast, when we have removed all excited bound states during the time propagation, the chirp dependence has disappeared. This is a clear evidence that the influence of the chirp on the photoionization processes essentially comes from the intermediate bound states. Consistently, when the photon energy is chosen to be larger than the ionization potential where single-photon ionization is the lowest order ionization process, we observe practically no chirp dependence.

Apart from the natural extension to the higher intensity range we have mentioned in the end of the previous section, another interesting and open question would be how the photoionization process is affected by the carrier-envelope phase of chirped laser pulses. For the case of a transformlimited pulse we have already answered this question in the multiphoton ionization regime $[14,15]$. For the case of chirped laser pulses we have recently found that the effects of the carrier-envelope phase is still very significant. We will report on our findings elsewhere in the near future.

\section{ACKNOWLEDGMENTS}

The author acknowledges Dr. X. Liu for bringing the importance of pulse chirp on the ionization dynamics to his attention. This work was supported by the Grant-in-Aid for scientific research from the Ministry of Education and Science of Japan. 
[1] P. Agostini, F. Fabre, G. Mainfray, G. Petite, and N. K. Rahman, Phys. Rev. Lett. 42, 1127 (1979).

[2] L. F. DiMauro and P. Agostini, Adv. At., Mol., Opt. Phys. 35, 79 (1995).

[3] Takashi Nakajima and Gabriela Buica, Phys. Rev. A 74, 023411 (2006).

[4] B. Chatel, J. Degert, S. Stock, and B. Girard, Phys. Rev. A 68, 041402(R) (2003).

[5] M. Wollenhaupt, A. Prakelt, C. Sarpe-Tudoran, D. Liese, and T. Baumert, Appl. Phys. B: Lasers Opt. 82, 183 (2006).

[6] R. Marani and E. J. Robinson, J. Phys. B 32, 711 (1999).

[7] S. Meyer, C. Meier, and V. Engel, J. Chem. Phys. 108, 7631 (1998).

[8] T. Frohnmeyer and T. Baumert, Appl. Phys. B: Lasers Opt. 71, 259 (2000).

[9] T. Lohmuller, M. Erdmann, and V. Engel, Chem. Phys. Lett. 373, 319 (2003).
[10] R. Itakura, K. Yamanouchi, T. Tanabe, T. Okamoto, and F. Kannari, J. Chem. Phys. 119, 4179 (2003).

[11] H. G. Breunig, A. Lauer, and K. M. Weitzel, J. Phys. Chem. A 110, 6395 (2006).

[12] X. Tang, H. Rudolph, and P. Lambropoulos, Phys. Rev. Lett. 65, 3269 (1990).

[13] P. Lambropoulos, P. Maragakis, and J. Zhang, Phys. Rep. 305, 203 (1998).

[14] Takashi Nakajima and Shuntaro Watanabe, Phys. Rev. Lett. 96, 213001 (2006).

[15] Takashi Nakajima and Shuntaro Watanabe, Opt. Lett. 31, 1920 (2006).

[16] T. N. Chang, Many-Body Theory of Atomic Structure and Photoionization (World Scientific, Singapore, 1993), Chap. 8.

[17] K. C. Kulander, Phys. Rev. A 38, 778 (1988).

[18] M. Aymar, J. Phys. B 11, 1413 (1978). 